\title{
Social E-Book Reader: Development of Interactional Model and Quality Analysis User Experience of E-Book Reader based Social Learning
}

\author{
Osiany Nurlansa, Ratna Wardani \\ Educational of Electronics and Informatics Engineering Master's Program, \\ Graduate School \\ Universitas Negeri Yogyakarta \\ Yogyakarta, Indonesia \\ osiany.nurlansa2015@student.uny.ac.id
}

\begin{abstract}
This research aims to develop and produce user interface recommendation of Social E-Book Reader application that can accommodate two-way interaction model and learning style in social learning according to user's habit and requirement tested with User Experience Questionnaire (UEQ) standard. The development design uses the Goal Direct Design (GDD) procedure consisting of (a) preliminary observation using the Educational Usefulness and User Interface Usability aspects of the Research phase, (b) making the product personality and user activity design in the Modeling stage, (c) based on the persona at the Requirement stage, (d) create the product framework at the Framework stage, (e) realize the application user interface in the mobile device in the Refinement stage, (f) and the feasibility test using UEQ at the Support stage. There are 10 book readers at the initial observation stage and 34 students as subjects on the feasibility test of interaction based on user experience. Data collection techniques used questionnaires, direct observation, and interviews. The data analysis technique uses UEQ standard calculation consisting of six scales with 26 items. Each item has scaled from -3 to +3 with Benchmark calculations, Confidence Interval values, correlation per item and Cronbach Alpha correlation. In this test, the processed data is from student ratings (book readers) to new interactions offered on Social EBook Reader. The results indicate that a new innovation in social interaction with Social E-Book Reader makes it more prominent in factors related to non-task related quality aspects or about the renewal of existing e-book readers (Hedonism).
\end{abstract}

Keywords-user experience, user interface, e-book reader, goal direct design, social learning, social e-book reader

\section{INTRODUCTION}

The potential of the Internet-based technology is as a supporting medium for human activities to do more and more practical activities. Based on research data from Indonesian Digital Association (IDA) supported by Baidu Indonesia and implemented by GfK global research institute, $96 \%$ of the highest smartphone users are in Indonesia [1]. Based on data from APJII of 2015 regarding the age of Internet users in Indonesia, the majority of users aged 18-25 years, occupying $49 \%$ of the total number of Internet users in Indonesia. That is, it can be said that the segment of internet users in Indonesia are those who belong to the category of digital natives.

Technological development and support have been able to bring up methods in the scope of individuals and institutions related to the way or model to perform an activity. One example in the field of education, the learning process is undertaken within the scope of the institution is no longer dependent on the teacher-centered but rather directed to the student-centered side. Coupled with Millennial or Y generation where this generation consists of a group of people born after generation $\mathrm{X}$, i.e., people born in the range of 1980-2000s. Based on the infographics from Commlab India, the learning strategies of Gen-Y include Just-in-time learning, Bite-sized learning or micro learning, collaborative and social learning, multi-device learning, and knowledge in multiple formats. This shows the readiness of the current era in achieving a goal or in solving problems through collaborative and social learning.

Schwartz in his website on Personalized Learning states that the infographic created by Mia MacMeekin indicates that reading is a profound activity, done anywhere and on the basis of their own willingness to reach high levels (advance). E-book one of the technological innovations to provide easy reading, get books from various sources anywhere and anytime. The current e-book is a computerized representation of a textbook containing text and images, or reflowable results reconstructed by software resembling pages on reading devices [2]. Technological advances coupled with the growing use of smartphones in Indonesia are an opportunity for the development of e-books and supporting applications more than they already exist today.

The survey results in a study stated that the main advantage of the e-book is that access is available anywhere and anytime [3]. The further development of the E-book as a medium of learning suggests that the features available in social media applications allow users to interact comfortably and unknowingly generate internal motivation for users in socializing [4]. Related to that, the e-book as a learning medium that is used as one of the current learning sources is said to have not provided a mechanism or a platform that 
allows accommodating the style of learning that is contemporary with the base of networked learning. The current e-book is static in the sense of minimal interaction that the platform can facilitate.

In the Usability of Hypermedia Educational e-Books research presents a number of evaluation criteria for e-books in the field of education and explains how the evaluation procedures in assisting developers in terms of education applications can evaluate existing applications and provide guidance in handling the requirements of educational application development during the development process. The criteria used are Educational Usefulness consisting of richness, completeness, motivation, hypertext structure, autonomy, competence, and flexibility; aspects of User Interface Usability consists of aesthetic, consistency, selfevidence, the naturalness of metaphors and predictability [5]. Based on preliminary observation, in each aspect is still less or need to improve better than e-book reader that already exists. The results of preliminary observations are shown in Figure 1.

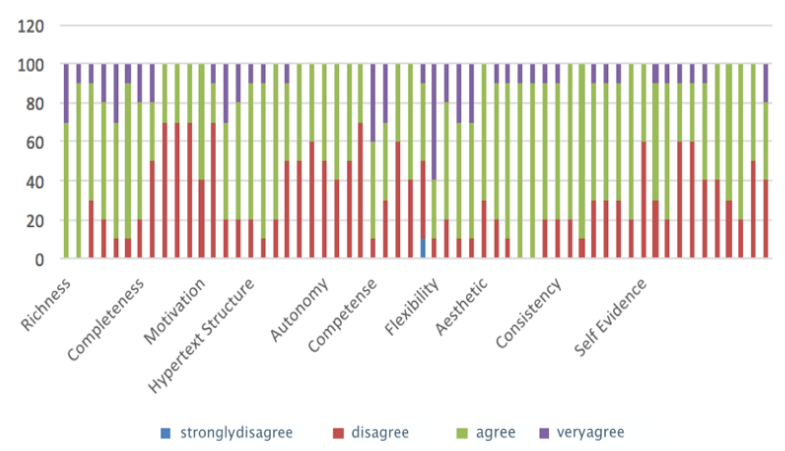

Fig. 1. Diagrams of observational results on educational usefulness and user interface usability aspects.

There are several issues to be outlined in this paper, namely:

- What kind of user interface recommendation can accommodate two-way interaction model and learning style in social learning on the social e-book reader?

- How is the recommendation quality of social e-book reader interface based on User Experience Questionnaire standard?

\section{METHOD}

\section{A. Research on the development of e-book reader in the field of education}

An interactive e-book as a means of developing teaching materials based on the mobile application has resulted in the model of interaction and design of e-book model as a platform using an object-oriented approach. The interaction model generated is a discussion forum in which each actor (learner) can interact with other actors to discuss or discuss one or more of the topics contained in the book being read. This approach can improve the effectiveness of the learning process through aspects of learning, sharing, and publishing [4]. However, in this research has not tested the model of interaction and model design on the user experience side, therefore in the research of social e-book reader, the researcher refers to the interaction model generated by Ratna Wardani research which then recommends user interface based on user experience and needs and tested based on user experience using UEQ [4].

The design of the e-book reader interface with the mobile user experience approach states that using the Goal Direct Design approach is appropriately used as a mockup design method of content display and e-book reader application features as it covers the steps of observing user behavior [6]. In research conducted by Dharma, the pragmatic aspect assessment is more prominent than Hedonist, as there is no significant renewal of existing ebook reader applications [6]. With the new add-in feature, it integrates the interaction model in social learning and e-book reader is expected to improve the Hedonis aspect in social ebook reader that will be developed.

\section{B. Research based on observation of e-book reader that exist}

In evaluating existing educational tools not only involves testing user interfaces but also didactic methods, learning materials, and interaction mechanisms to prove whether existing educational tools help users achieve their goals for learning. In his report, Paloma mentioned the evaluation criteria to explore in order to input further development or can be called the needs analysis during the design process using evaluation procedures on two factors namely educational usefulness and user interface usability [5].

In the initial observations made on both these factors for e-book reader applications already in the field and used by the user stated that the e-book reader is currently reviewed based on aspects of Educational Usefulness on the Richness factor the user wants the e-book reader to provide more information how e-books are rich in useful features, how presentation styles and e-book interactions can meet the needs of the user's style of learning. In the Completeness factor of how e-books can assist users in completing tasks, users can mark and annotate important notes in e-books, users can edit important content in e-books, through e-book users can interact with other users who have the same interests, in the e-book, users can find discussion groups. In Motivation factor users want to be able to find the exercise questions to assess their own ability after reading the e-book, the user finds the key answer from the exercise problem, can support in learning and easy to use. In the Hypertext Structure factor the user wants to connect to related pages via hypertext, the module structure in the e-book is standardized so that it can improve the usability and ease of maintenance, the user is easy to use navigation when accessing the e-book, the structure in hypertext is easy to use, content on hypertext is already balanced. In the Autonomy factor, the user wants the navigation and interaction in the e-book easy to use, the user can freely interact with both the application and with other users, the e-book already gives instructions and directs to the help center when users feel confused, the user gets the appropriate level of choice with his ability. In the Competence factor hope users can feel the e-book read useful, the user can easily use the e-book as needed, the ease of finding the help menu, the user understand the contents of the content in the help menu, the user understands the contents of the content in the help menu, and e-book can customize user needs. On the Flexibility factor, the user feels 
it is appropriate so that it will not be removed and still maintained for further development.

The current e-book reader is reviewed based on the aspect of User Interface Usability on the desired Aesthetic factor to be further improved based on user requirements, i.e., the user can adjust the size and type of font as desired. Consistency factors are emphasized on improving the laying and proper functioning of small notes, marking important information and sorting the laying of frequently used icons in strategic locations and easy to find. In the Self-Evidence factor the user needs terms that are used familiarly to the user, the user can find the synopsis before choosing the ebook to read, find the multimedia content (consisting of text, image, sound, and video) in e-book, link in e-book allows users to access information outside of e-books, users easily find the intended content through the list of contents are linked, and error messages in the popup can be easily understood the user. In the Naturalness of Metaphors factor, users can use e-books to read comprehensively, and the user can complete more work than is commonly used. In the Predictability factor, users want to be able to find synopsis easily before opening or selecting e-books to read. The results of this study underline that the current e-book is still a minimal feature to be able to interact in two directions, and if the researcher adds one sharing feature an e-book reader that will be made, it is expected the hedonic and pragmatic aspects will be above average.

\section{Research is reviewed through the use of Goal-Directed Design (GDD)}

Goal-Directed Design combines ethnographic techniques, stakeholder interviews, market research, user model details, basic design scenarios, and some interaction principles. this method provides solutions that meet user needs and goals. The Goal-directed design process is divided into 6 steps according shown in Figure 2

\begin{tabular}{|c|c|c|c|c|c|}
\hline $\begin{array}{c}\text { Research } \\
\text { users and } \\
\text { the domain }\end{array}$ & $\begin{array}{l}\text { Modeling } \\
\text { of users } \\
\text { and use } \\
\text { context }\end{array}$ & $\begin{array}{c}\text { Requirements } \\
\text { definition of } \\
\text { user, business, } \\
\text { and technical } \\
\text { needs }\end{array}$ & $\begin{array}{l}\text { Framework } \\
\text { definition of } \\
\text { design } \\
\text { structure } \\
\text { and flow }\end{array}$ & $\begin{array}{l}\text { Refinement } \\
\text { of behaviors, } \\
\text { form, and } \\
\text { content }\end{array}$ & $\begin{array}{l}\text { Support } \\
\text { developmen } \\
\text { needs }\end{array}$ \\
\hline
\end{tabular}

Fig. 2. Stage on Goal-Directed Design [7].

The initial phase of the task-oriented method analyzes the user's activities, first aiming to get a typical user activity when traveling. Both ways users make decisions, information needed to make decisions, and how users get information. The task-oriented concept is suitable for situations where users do not have to be disturbed in their normal activities, so the observation is more natural. One approach to complementing this problem is the "Experience Clip" technique, UX techniques on user activity when on the road. The user-centered design for mobile device design to achieve strong UX [8]. The design focus is always not out of usefulness, not only its usability.

Edelson explains that research in the field of education increasingly uses design as a means of promoting understanding [9]. The design research paradigm emerges to treat design as a strategy for developing and refining theory. Three types of theories can be developed through design research: domain theory, design frameworks, and design methodologies. In his article also presents examples of research programs investigating software supports for reflective inquiry. Daniel argues for design research as a form of educational research because (1) the design offers an opportunity to learn a unique lesson, (2) practical design study results that can be directly applied, and (3) research designs involved researchers in direct improvement practice education. Icek Ajzen and Thomas J Madden used the Goal Direct Design method to understand attitudes and predict social behavior [10].

The results of this study underline that the use of Goal Direct Design method can recommend the social e-book reader application interface to suit the needs and user experience.

\section{Research is reviewed from User Experience Questionnaire (UEQ) usage}

Use of international software products, it is important to know the culture, language, and behavior of citizens. In order to evaluate the product, it is important to know how people from a country behave and express their feelings [11]. In evaluating, Cota uses UEQ as a questionnaire to measure the success rate of a designed product. Cota revealed that UEQ allows a quick assessment of the user experience for any interactive product. The questionnaire scale is designed to include a thorough impression of the user experience. The questionnaire format supports direct user feedback to express feelings, impressions, and attitudes that arise when they use the product. In addition, this questionnaire is short enough to be applied as an online form. This can be filled in by participants within minutes even if the demographic question is added at the beginning or end of the questionnaire. The validation results show that the questionnaire scale is consistent and can measure the quality of the desired product.

In a study conducted by Martin Schrepp et al. also mentioned that in evaluating user experience, using UEQ is the best choice [12]. In his research entitled 'Applying the User Experience Questionnaire (UEQ) in Different Evaluation Scenarios' mentions that Good user experience is essential for the success of interactive products. Improving product quality, it's also important to measure the user experience in an efficient and reliable way. Different questions can be the reason behind the desire to quantify a user's experience of a product quantitatively. Some typical questions related to measuring user experience and showing how these questions can be answered with questionnaires with relatively low effort. This study uses a UEQ user experience questionnaire, but the general approach can be transferred to other questionnaires as well.

User Experience Questionnaire (UEQ) provides tremendous benefits. The UEQ provides a comprehensive impression of user experience, ranging from classic usability aspects and aspects of the user experience. Any evaluation effort to measure usability is usually taken from the student's perspective. This problem is very important because the quality of the use of the learning management system will affect student learning performance while learning online. The aims of this study are: (1) to understand the user experience of students when using learning management systems in Computer Science education; (2) to apply a multimethod approach in evaluating the student user experience; and (3) provide recommendations to improve the learning management system [13]. 
In UEQ each item has a semantic differential form, i.e., each item is represented by two terms with the opposite meaning. The random sequence of terms is per item, i.e., half of the items on the initial scale with positive terms and the other half of the items start with a negative term [14]. UEQ structure consists of as in Figure 3

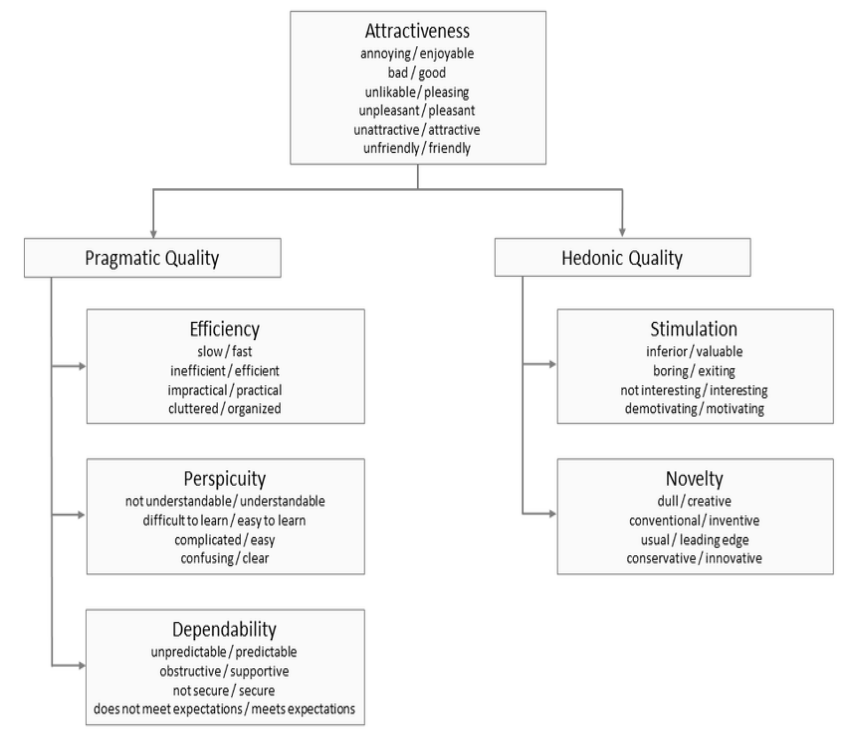

Fig. 3. The structure of the UEQ assumption scale (Http://www.ueqonline.org/)

It underlies this research that using UEQ to evaluate the social e-book reader application interface can be easy, efficiently and quickly see the result of interfaces built so that the resulting interface can get a positive value, feasible and meet the pragmatic and hedonic aspects of users.

\section{RESULT AND DISCUSSION}

\section{A. Design of the Development Model}

The development procedure that is carried out to produce the social e-book reader application-based Smartphone product interface begins with the pre-survey stage. The goal is to observe the habits and needs of users who will be used as key features and interactions on the discussion of products to be developed. The instruments used are interview and observation. The next stage is done with Goal Direct Design development model. The stages are:

\section{1) Research}

Observation of habits taken at places that have a lot of interaction with the book, the bookstore, and library, and observe the habits of users when discussing and interacting both offline and online.

\section{2) Modeling}

After obtaining interview and observation data, this stage serves to decide the design of activities and persona. Activity design is the main user activity category, such as how users search for books, ask staff, discuss with friends and so on. The data obtained will be reinforced by reference research on the reader of the book. Persona formation is the stage of collecting user data by cluster sampling method to be interviewed. The results of the interview will be used as a basis for mapping the same habits of each user. Then proceed by using the behavior variable method to group the same habits, this is useful for determining the persona of social e-book reader mockup product.

After getting the variable for the mapping persona, the interview result of the respondent will be included in each variable. Galih, Yusinta, Pinki, Yeni, Vivy, Nugroho and Santi are the respondents entered on the product persona. Seen in Figure 3 there are some respondents who have similarities in almost several variables

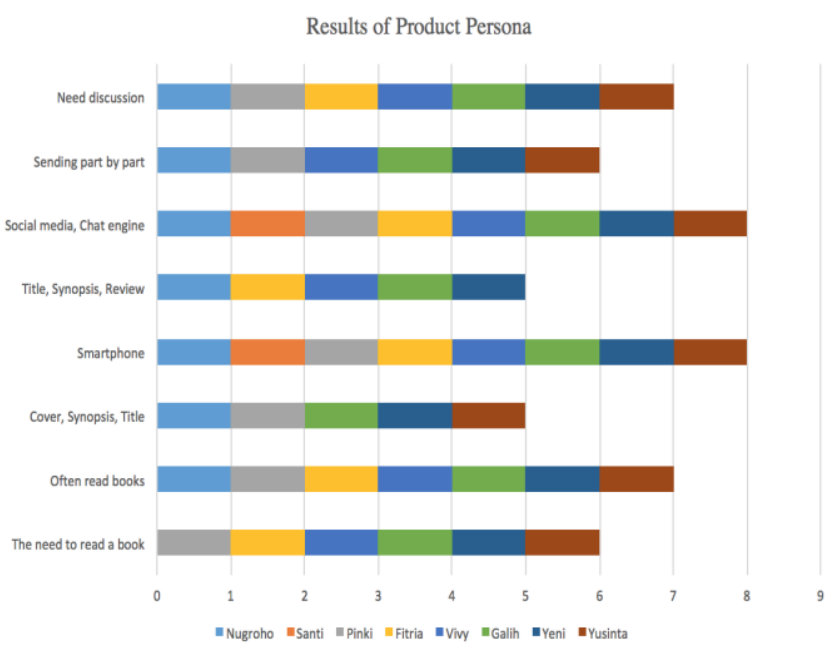

Fig. 4. Results of product persona for the development of Social E-book Reader

\section{3) Requirement}

This phase focuses on the details of the goals and needs of the users in accordance with the persona that is formed. This Persona facilitates the determination of styles, details and design interactions that match the persona's behavior. The results are activity scenarios and persona needs. At this stage, a combination of research results, user habits observations and the results of the formation of persona to meet the needs of the mockup. Fulfillment is a mockup feature that fit the habits and needs of users. At this stage, the disclosure of features required by users is also assisted with the results of early observation on aspects of Educational Usefulness and User Interface Usability.

\section{4) Framework}

This stage undertakes the overall creation of the product framework, defining the basic framework for product behavior and visual design. The basis for making this visual design is the persona data and user activity observation. User questions identify the needs of the required features. The searched and viewed object is the content of each application page. Observation of user activities is used as the basis for the division of application pages.

\section{5) Refinement}

Mockup creation stage is the realization stage of interaction design in the form of user interface. The interaction design that has been obtained at the design stage is used as the foundation for making this mockup. Persona as the main user of this application, used as a model for making features, objects, and functions incorporated in the user's face to face so it will be as expected. In this research, the interface will be built on Marvell applications to be used in various smartphone devices. The results of observation, persona, and framework are made whole into one. The social 
e-book reader interface is embodied in a mobile device, accessible and has an action button. Users can experiment on product interactions, as well as information obtained on the product.

\section{6) Support}

UEQ assessment is used for user evaluation of mockup built applications. Application evaluation includes interaction testing built on a user interface, functionality, and conformity to user requirements. Another assessment is to judge the look and feel mockup. To find out the results of the assessment, the mockup will be tested using the UEQ that represents the interaction, feelings of use and function when using the mockup. Overall, ongoing evaluation, the continuous evaluation from the beginning to the end of the product development process so that the product can achieve a decent quality according to UEQ indicator.

\section{B. UX Assessment Results}

TABLE I. THE RESULTS OF THE QUESTIONNAIRE SHOWED THE RESULTS OF THE ASSESSMENT AFTER DATA TRANSFORMATION

\begin{tabular}{|c|c|c|c|} 
Scale & Mean & Comparison to benchmark & Interpretation \\
\hline Attractiveness & 1.87254902 & Excellent & In the range of the $10 \%$ best results \\
\hline Perspicuity & 1.816176471 & Good & $10 \%$ of results better, $75 \%$ of results worse \\
\hline Efficiency & 1.360294118 & Above Average & $25 \%$ of results better, $50 \%$ of results worse \\
\hline Dependability & 1.786764706 & Excellent & In the range of the $10 \%$ best results \\
\hline Stimulation & 1.926470588 & Excellent & In the range of the $10 \%$ best results \\
\hline Novelty & 1.823529412 & Excellent & In the range of the $10 \%$ best results
\end{tabular}

Based on UEQ Benchmark calculations on all scales of variables in social e-book reader shows that mean> 0.8 means that each scale in each variable has a positive value, with detail as follows: Attractiveness of 1.873 included in the Excellent category, Perspicuity of 1.816 included in the category of Good, Efficiency of 1,360 included in the category Above Average, Dependability of 1.787 included in the Excellent category, Stimulation of 1.926 included in the Excellent category, and Novelty of 1.824 included in the Excellent category.

UX Assessment focuses on Attractiveness related to Pragmatic and Hedonist factors. Pragmatic factors include Perspicuity (clarity), Efficiency (efficiency), and Dependability (accuracy). Meanwhile, the factors Hedonis include Stimulation (stimulation) and Novelty (novelty). From the results of the assessment on the table (number how) is the Pragmatic and Hedonic Quality table, the social e-book reader UX obtained that Hedonic Quality has the highest value of 1.88 , this shows that the design of social e-book reader interface is more prominent in related factors with non-task related quality aspects or about the renewal motivation of the current ebook reader.

\section{Confidence Interval Value Result}

TABLE I. CONFIDENCE INTERVAL VALUE RESUlT

\begin{tabular}{|c|c|c|c|c|c|c|}
\hline \multicolumn{7}{|c|}{ Confidence intervals $(\mathbf{p}=\mathbf{0 . 0 5})$ per scale } \\
\hline Scale & Mean & Std. Dev. & $\mathbf{N}$ & Confidence & \multicolumn{2}{|c|}{ Confidence interval } \\
\hline Attractiveness & 1.873 & 0.492 & 34 & 0.166 & 1.707 & 2.038 \\
\hline Perspicuity & 1.816 & 0.697 & 34 & 0.234 & 1.582 & 2.051 \\
\hline Efficiency & 1.360 & 0.767 & 34 & 0.258 & 1.103 & 1.618 \\
\hline Dependability & 1.787 & 0.812 & 34 & 0.273 & 1.514 & 2.060 \\
\hline Stimulation & 1.926 & 0.595 & 34 & 0.200 & 1.726 & 2.127 \\
\hline Novelty & 1.824 & 0.724 & 34 & 0.243 & 1.580 & 2.067 \\
\hline
\end{tabular}

In this trial, the probability used in Confidence Interval per item is 0.05 which means the error rate in this research is 0,05 . The Confidence Interval result table with 0.05 shows all the question items can be trusted for $95 \%$. The average result value in the mean column enters between the lower limit value and the upper limit of Confidence Interval. This means that the average response sample represents the average population on the item.

The result table of the questionnaire in the mean column shows the average value per variable. The mean value in all variables in the mean column is between the lower boundary value and the upper limit of Confidence Interval. this indicates that each variable can be trusted at $95 \%$. The average answer represents the average population in each variable. Assessment of respondents amounting to 34 represents the population for each item and variable can be trusted with equal to 0.05 .

\section{Correlation Assessment Results by Item and Coefficient of Cronbach Alpha}

The results of this stage are obtained from the measurement of the correlation between each item question. The item of the question represents the UX variable. The first assessment is the assessment of the Attractiveness variable which has 6 question items. Each question item is measured by its correlation. The value of the correlation coefficient (R) has a value boundary of at least -1 and at most 1 . According to Cronbach the requisite item requirement of each valid and reliable variable if it is $>0.6$.

TABLE II. CORRELATION ASSESSMENT RESUlTS By ITEM AND COEFFICIENT OF CRONBACH ALPHA
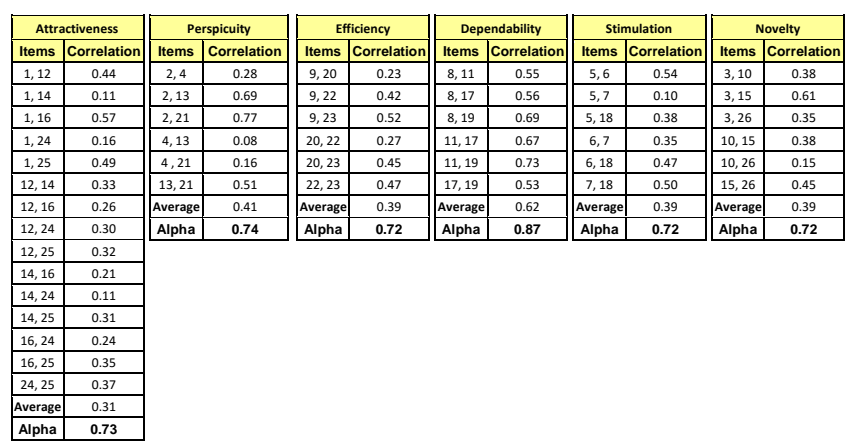

In Table 3, Correlation Value per Item and Cronbach Alpha Coefficient of Attractiveness or Attractiveness Variables in the items column, each line indicates 2 items measured by their correlation. All values between items have a positive value. So it can be said that the question items have a positive correlation. The question items are used to measure the general impression of the social e-book reader application interface and also to find out whether or not users like the social e-book reader application interface built. The result of Cronbach Alpha value on valid and reliable attractiveness variable is 0.73 .

Correlation Value per Item and Coefficient Cronbach Alpha variable Perspicuity or Clarity is obtained from 4 question items. All values between items have a positive value. So it can be said that the question items have a positive correlation. The question items are used to measure 
the social e-book reader application interface whether it is easy to understand and recognize. The value of Cronbach Alpha results in invalid and reliable variable perspicuity of 0.74 .

Correlation Value per Item and Coefficient of Cronbach Alpha Efficiency or Efficiency variable is obtained from 4 question items. All values between items have a positive value. So it can be said that the question items have a positive correlation. The question items are used to measure the fast and clear social e-book reader application interface from the user's point of view. The result of Cronbach Alpha value on valid and reliable Efficiency variable is 0.72 .

Correlation Value per Item and Coefficient Cronbach Alpha variable Dependability or Accuracy is obtained from 4 items of the questions. All values between items have a positive value. So it can be said that the question items have a positive correlation. The question items are used to measure user expectations of the social e-book reader application interface. The result of Cronbach Alpha value on valid and reliable Dependability variable is 0.87 .

Correlation Value per Item and Cronbach Alpha Coefficient of Stimulation or Stimulation variable are obtained from 4 question items. All values between items have a positive value. So it can be said that the question items have a positive correlation. Question items are used to measure user interest in using social e-book reader application interface. The result of Cronbach Alpha value on valid and reliable Stimulation variable is 0.72

Correlation Value per Item and Coefficient Cronbach Alpha Novelty or Novelty variable is derived from 4 question items. All values between items have a positive value. So it can be said that the question items have a positive correlation. Question items are used to measure creative product design, innovative, and attract the attention of users. The result of Cronbach Alpha value on Novelty variable is valid and reliable equal to 0.76

The value of each variable in the Cronbach Alpha test above 0.6 proves that the questionnaire items are valid and reliable.

\section{CONCLUSION}

Based on the research that has been done in the development of interaction model on the user interface of social e-book reader based on user experience, it can be concluded as follows:

This research produced recommendations of User Interface e-book reader application based on a Smartphone that can accommodate two-way interaction model and learning style in social learning. The interaction model generated based on preliminary observation uses the Educational Usefulness and User Interface Usability aspects to observe the User Experience on an existing e-book reader. The User Interface is built on the Goal Direct Design (GDD) method and is simulated via the Marvell platform. Stages of Goal Direct Design include Research, Modeling, Requirements, Framework, Refinement, and Support.

The Social Interface e-book reader user is testing using a quality standard of interaction at the International level of User Experience Questionnaire (UEQ). The results showed that the interaction on the user interface in terms of user experience testing based on UEQ standard has a positive value with the detail of Attractiveness factor quality of 1.873 including Excellent category, Perspicuity of 1.816 including Good category, Efficiency of 1,360 including Above Average category, Dependability of 1,787 including Excellent category, Stimulation of 1,926 including Excellent category, and Novelty for 1,824 exclusive category. The result of Pragmatic and Hedonic Quality, Social E-Book Reader, found that Hedonic Quality has the highest value of 1.88 , it shows that the user interface of Social E-Book Reader is more prominent in factors related to non-task related quality aspects or about the renewal of e-book reader that currently exists.

\section{REFERENCES}

[1] Mailanto, A. (2016, Maret 16). 96 Persen Pengguna Smartphone Tertinggi Ada di Indonesia. Retrieved September 20, 2016, from OkezoneTechno:

https://techno.okezone.com/read/2016/03/16/207/1337776/96-persenpengguna-smartphone-tertinggi-ada-di-indonesia

[2] Carden, M. T. (2008). E-books are not books. Conference on Information and Knowledge Management. Proc. 2008 ACM Work (pp. 9-12). B. Repos.: Res. Adv. large Digit.

[3] Muir, L. and Hawes, G. (2013). The case for e-book literacy: undergraduate students' experience with e-books for coursework. Journal of Academic Librarianship, 39(3), pp. 260-274.

[4] Wardani, Ratna. (2015). E-Book Interaktif Sebagai Sarana Pengembangan Bahan Ajar Berbasis Mobile Application. Laporan Penelitian Fundamental 2015. LPPM UNY

[5] Díaz, P. (2003). Usability of hypermedia educational e-books. D-Lib magazine, 9 (3), 564-568.

[6] Surya, Dharma. (2015). Perancangan Antarmuka E-Book Readerdengan Pendekatan MobilityUser Experience. Tesis. Universitas Gajah Mada. Yogyakarta

[7] Cooper, A., Reimann, R., Cronin, D., \& Noessel, C. (2014). About Face. The Essentials of Interaction Design. Wiley: John Wiley \& Sons, Inc.

[8] Harrison, C., Medrington, S., \& Stran, W. (2011, 4 30). User Centred Design Research Methods for Mobile Industry Practitioners. Journal of Mobile Media.

[9] Edelson, D. C. (2002). Design Research: What We Learn When We Engage in Design. THE JOURNAL OF THE LEARNING SCIENCES, 11 (1), 105-121.

[10] Ajzen, I., \& Madden, T. J. (1986). Prediction of goal-directed behavior: Attitudes, intentions, and perceived behavioral control. Journal of Experimental Social Psychology, 22 (5), 453-474.

[11] Cota, M. P., Thomaschewski, J., Schrepp, M., \& Gonçalves, R. (2014). Efficient Measurement of the User Experience. A Portuguese Version. 5th International Conference on Software Development and Technologies for Enhancing Accessibility and Fighting Infoexclusion, DSAI 2013.27 , pp. 491 - 498. Procedia Computer Science.

[12] Schrepp M., Hinderks A., Thomaschewski J. (2014) Applying the User Experience Questionnaire (UEQ) in Different Evaluation Scenarios. In: Marcus A. (eds) Design, User Experience, and Usability. Theories, Methods, and Tools for Designing the User

[13] Santoso, H., Schrepp, M., Isal, R. K., Utomo, A. Y., \& Priyogi, B. (2016). Measuring User Experience of the Student-Centered eLearning Environment. The Journal of Educators Online-JEO, 13 (1), 58-79.

[14] Schrepp, M. (2015, September 28). User Experience Questionnaire Handbook. Retrieved Oktober 4, 2016, from ResearchGate: https://www.researchgate.net/publication/281973617_User_Experienc e_Questionnaire_Handbook 